\title{
Analysis of a Wearable Robotic System for Ankle Rehabilitation ${ }^{+}$
}

\author{
Matteo Russo ${ }^{1}(\mathbb{D})$ and Marco Ceccarelli ${ }^{2, *}$ \\ 1 The Rolls-Royce UTC in Manufacturing and On-Wing Technology, Faculty of Engineering, \\ University of Nottingham, Nottingham NG8 1BB, UK; matteo.russo@nottingham.ac.uk \\ 2 LARM ${ }^{2}$ : Laboratory of Robot Mechatronics, University of Rome "Tor Vergata", 00133 Rome, Italy \\ * Correspondence: marco.ceccarelli@uniroma2.it \\ + This work is an expansion of a paper presented at The 3rd International Conference of IFToMM Italy \\ (IFIT 2020): Russo, M., Ceccarelli, M. A wearable device for ankle motion assistance. In Advances in Italian \\ Mechanism Science: Mechanisms and Machine Science 91, Springer, Cham, Switzerland, 2020 (in print).
}

Received: 10 August 2020; Accepted: 26 August 2020; Published: 27 August 2020

check for updates

\begin{abstract}
As one of the most commonly injured joints of the human body, the ankle is often subject to sprains or fractures that require motion assistance to recover mobility. Whereas physiotherapists usually perform rehabilitation in one-on-one sessions with patients, several successful robotic rehabilitation solutions have been proposed in the last years. However, their design is usually bulky and requires the patient to sit or stand in a static position. A lightweight wearable device for ankle motion assistance, the CABLEankle, is here proposed for motion ankle exercising in rehabilitation and training. The CABLEankle is based on a cable-driven S-4SPS parallel architecture, which enables motion assistance over the large motion range of the human ankle in a walking gait. The proposed mechanism design is analyzed with kinematic and static models, and the force closure workspace of the mechanism is discussed with analytical results. Finally, the feasibility of the proposed design is investigated through numerical simulations over the ankle motion range as a characterization of the peculiar motion.
\end{abstract}

Keywords: service robotics; medical devices; rehabilitation; parallel manipulators; cable-driven robots

\section{Introduction}

The ankle joint is critical to locomotion by sustaining the load of the whole body while exerting key forces during push-off, leg swing, and center of mass advancement during the human gait [1]. Unfortunately, it is also one of the most commonly injured joints of the human body, often subject to sprains or fractures that hinder or impede a full walking capability of a patient [2]. In order to recover full mobility, rehabilitation and motion assistance are required [3]. Rehabilitation training is usually performed by a physiotherapist in one-on-one sessions with a patient because it requires the physiotherapist's full attention.

Robot-assisted rehabilitation was proposed as an efficient alternative, since it would enable a single physiotherapist to assist multiple patients at the same time, potentially teleoperating the robots from a remote location. The feasibility of a robotic solution for the assistance of patients in the recovery of gait functions has been proved by several research groups [4-7]. However, most of the ankle rehabilitation robots are based on static platform designs, as illustrated in [8-16]. In the work in [8], rehabilitation is performed with the patient standing on two platforms with variable orientation. This solution requires the patient to be able to stand up, and it is characterized by a bulky (non-portable) device. Another grounded design is proposed in [9] for the rehabilitative exercise of a single leg, which is similar to the prototype in [10] with a mechanism driven by pneumatic muscles. An evolution 
of the same design is reported in [11]. Smaller but still grounded devices are introduced in [12] and [13] as based on a high-performance parallel architecture and a compliant mechanism, respectively.

All these robots are characterized by static designs, and most of them, such as the prototypes in [14-16], are fixed to the ground or are difficult to move due to a large footprint and weight. In order to overcome this limit, few research groups have proposed wearable designs such as ankle exoskeletons and orthoses [3,17]. These wearable rehabilitation systems are usually characterized by rigid or compliant links and bulky bodies, thus still requiring additional support for their significant weight.

Cable-driven robots may solve all these issues thanks to their lightweight structures. Additionally, they can be safer than conventional mechanisms thanks to their negligible moving mass [18]. Portable cable-driven rehabilitation robots have already been introduced in [18-20] for legs and elbows. In fact, cable-driven parallel mechanisms have recently attracted attention even in specific applications, as reported in the dedicated conference series in [21], where nevertheless the topic of ankle assistance devices is not analyzed. Only a few research groups have proposed static (non-portable) designs for ankle rehabilitation [22,23]. In particular, the work in [22] studies the feasibility of cable-driven ankle rehabilitation without proposing a specific design. A grounded platform is described in [23], but its four cables are not enough to enable full control of the ankle joint, since the proposed device is fixed to the foot but not to the shank of the patient. While three degrees of freedom is enough to control the foot motion with respect to the leg, grounded designs require either more degrees of freedom or additional constraints to the leg. According to the authors' knowledge, there is no portable cable-driven solution available for ankle motion assistance.

The CABLEankle is presented in this paper as a lightweight wearable robot for ankle motion assistance with a cable-driven architecture. The CABLEankle is inspired by the CADEL architecture first presented in [20], which can be modeled as a cable-driven S-4SPS parallel mechanism. The original elbow rehabilitation mechanism is adapted to the new geometric and motion requirements for ankles. In [24], the conceptual design of the CABLEankle is briefly introduced with its kinematic model and a numeric simulation for a limited two-degree-of-freedom motion range. This manuscript expands the research work of [24] with a detailed kinematic and static analysis that includes a novel formulation for the force-closure model of the proposed cable-driven mechanism. Furthermore, a numerical simulation is included to validate the feasibility of the CABLEankle for the full ankle mobility range, in order to characterize the performance of this novel design in terms of motion range, cable tension, load on the ankle joint, and motor power.

\section{Materials and Methods}

In this section, the design process of the proposed ankle rehabilitation device is reported with the main methods used to synthesize and analyze the mechanism. First, motion requirements are defined by studying the biomechanics of the human ankle joint, with a focus on its mobility. Then, the conceptual design of the mechanism is introduced with its main characteristics. A closed-form analytical solution is provided for the kinematics of the proposed device, which is needed to actuate and control the ankle motion. A static model is then reported to evaluate cable tension and ankle joint reaction forces during motion. Finally, a novel formulation for force-closure of cable-driven mechanisms is also presented in this paper to validate the feasibility and the performance of the device in its workspace.

\subsection{Motion Requirements from Ankle Biomechanics}

The ankle joint complex connects the lower leg and the foot, and it is made up of three main joints, namely the talocalcaneal, tibiotalar, and transverse-tarsal joint. It can sustain large compressive and shear forces while keeping a high degree of stability during gait [25]. The complex mobility of the ankle can be approximated by its three main motion modes, which are illustrated in Figure 1: plantarflexion and dorsiflexion, in the sagittal plane; abduction and adduction, in the transverse plane; inversion and eversion, in the frontal plane. 


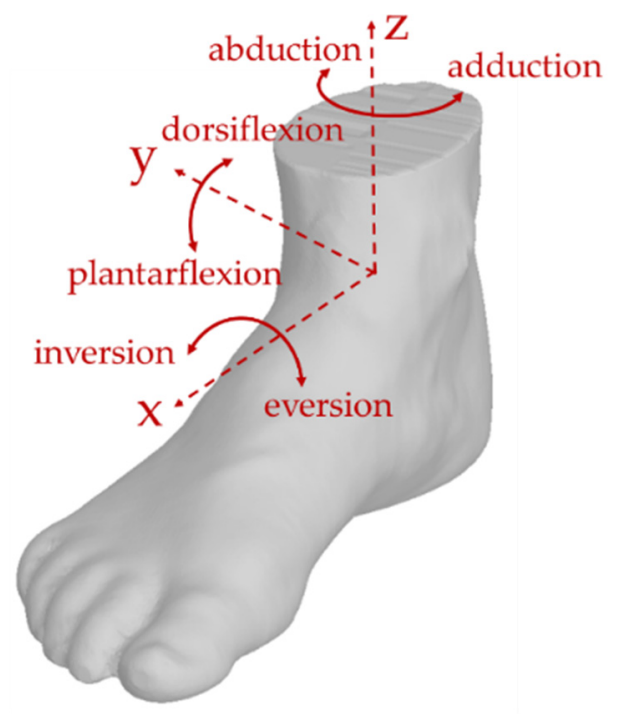

Figure 1. Morphological motions of the human ankle joint.

The ranges of motion of these modes are characterized by a significant variability between individuals, due to geographical/cultural differences, anatomical structures, and distinct data-acquisition methodologies. In order to define a target range of motion for an ankle-assisting device, conservative values for the ankle range of motion as per Figure 1 have been extracted from [25-27], as summarized in Table 1 referring to daily activities.

Table 1. Ranges of motion of the human ankle joint [25-27].

\begin{tabular}{ccccc}
\hline Motion & Dorsiflexion & Plantarflexion & Abduction/Adduction & Inversion/Eversion \\
\hline Range limits & $20 \mathrm{deg}$ & $50 \mathrm{deg}$ & $\pm 10 \mathrm{deg}$ & $\pm 12 \mathrm{deg}$ \\
\hline
\end{tabular}

Design requirements may refer to healthy ranges of motion as in Table 1, but for motion assistance smaller ranges can be considered mainly in application for rehabilitant therapies and elderly people assistance. In addition to the kinematic aspects of the range of motion, requirements for design and operation purposes can be considered in terms of actions on the ankle to achieve a desired ankle movement either in full passive mode or with a user participation that can be controlled and guided. This action can be sized within a range of 1 to $20 \mathrm{~N}$ when considering unloaded ankle motion in the air and can be scaled up to more than $100 \mathrm{~N}$ if users stand up on the foot. In general, however, a rehabilitation therapy can be planned with a leg in the air. Therefore, the force requirements for the device developments can be assumed as less than $30 \mathrm{~N}$, considering also possible exercise training to reinforce the ankle muscular system.

\subsection{Conceptual Design of a Wearable Device for Ankle Motion Assistance}

Figure 2a shows a conceptual design of CABLEankle, a wearable device for ankle motion assistance. The above-mentioned issues are addressed with the indication of motion directions and with a scheme of a cable-driven system that is equipped also with suitable sensors (S) for monitoring and controlling purposes. The application of the device can range from motion guidance, as per exercise and augmentation of ankle capacity, to motion assistance, as per rehabilitation and physiatry ankle medical therapy. 


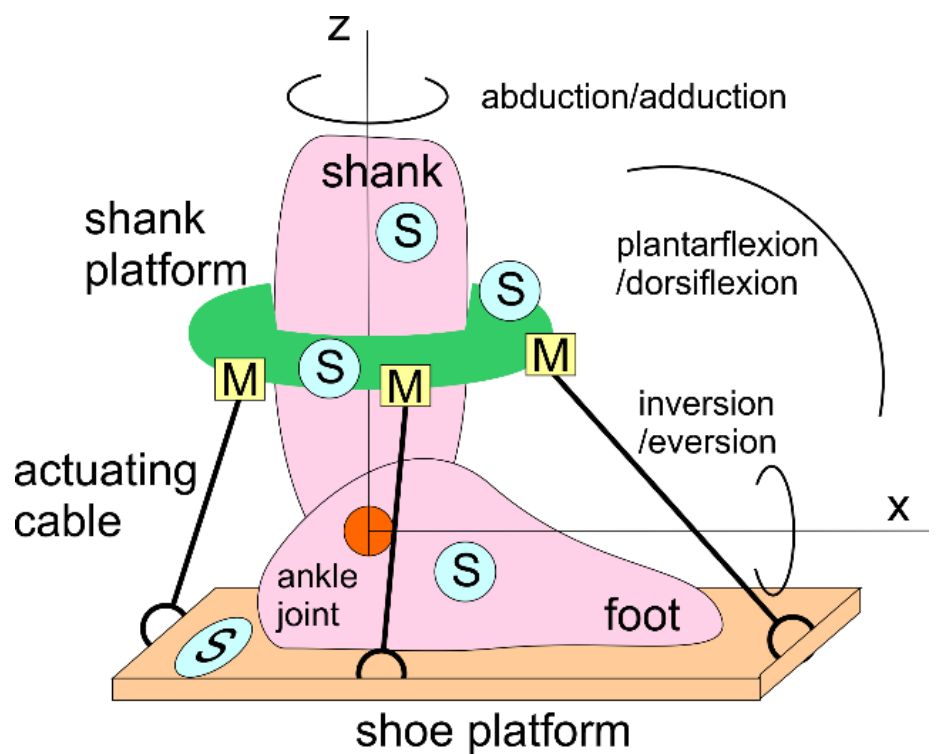

(a)

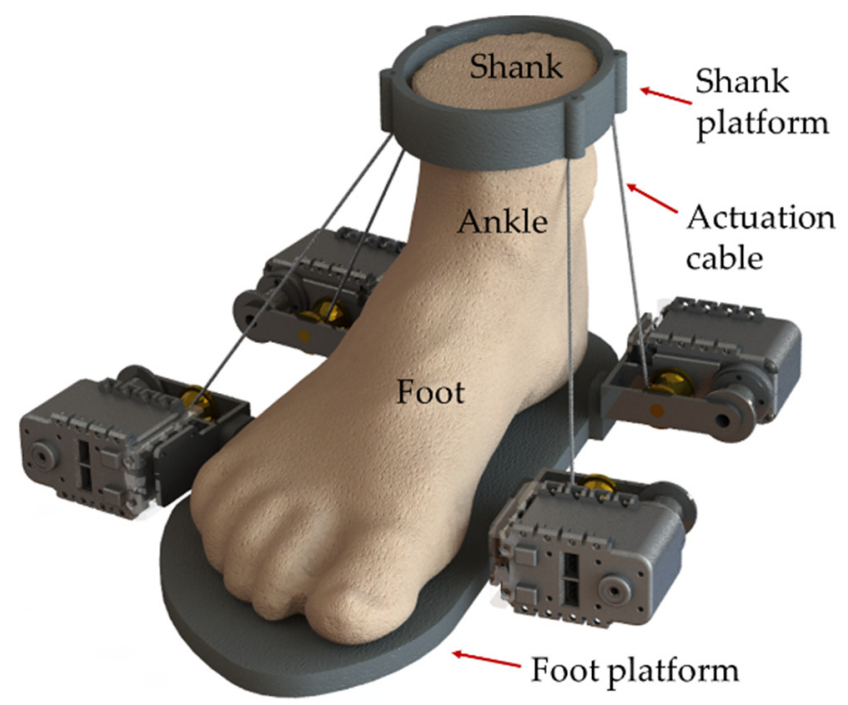

(b)

Figure 2. Proposed solution for ankle motion assistance: (a) conceptual design of the CABLEankle with main components (M: motors, S: sensors); (b) A CAD design for the proposed conceptual scheme.

The motion of the ankle with respect to the shank (part of a person's leg between the knee and the ankle) is planned by the relative motion of a foot platform, which can even include a shoe for wearing the system, with respect to a shank platform, which can be ring-shaped to be worn on the shank. The motors $(\mathrm{M})$ increase or decrease cable lengths to guide this relative motion with a mechanical structure that is equipped of a pulley and servomotor. The control system can use information from sensors (S), which can be both on board of the device and worn by the patient. These sensors can include accelerometers and IMUs, which enable gait analysis as reported, for example, in [28]. Sensors (S) can be used not only for operation control of the device, but also for monitoring of ankle medical issues, such as for measures of temperature, muscle reaction and blood pressure, as required in the device application. Figure $2 \mathrm{~b}$ shows a CAD design of a mechanical solution, highlighting the lightweight compact features of the conceptual design for portability and user-oriented operation. A power supply can also be integrated inside the foot platform, on the shank platform or in a box nearby the device. 


\subsection{Kinematic Analysis}

The proposed solution in Figure 2 is a cable-driven parallel mechanism that is inspired by the CADEL design for elbow rehabilitation in [20]. The device is characterized by a foot platform and a shank platform that are connected by four cables of varying length, as shown in the models of Figure 2. The two platforms are connected to the foot and the shank of the patient, respectively, by means of straps or equivalent comfortable means, and both of them can be assumed to be fixed to a patient's leg and foot. Thus, by varying the length of the cables, it is possible to activate a relative motion between foot and shank, guiding the patient's ankle motion. The kinematic behavior of the ankle motion can be approximated by a spherical joint with limited angular motion. Therefore, it is possible to model the proposed solution as a lower-mobility parallel mechanism with three degrees of freedom, by using a S-4SPS architecture, as shown in the model of Figure 3.

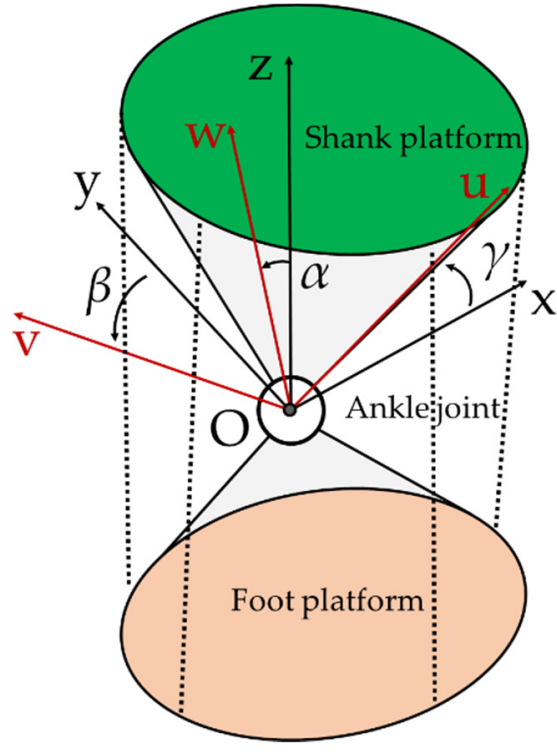

(a)

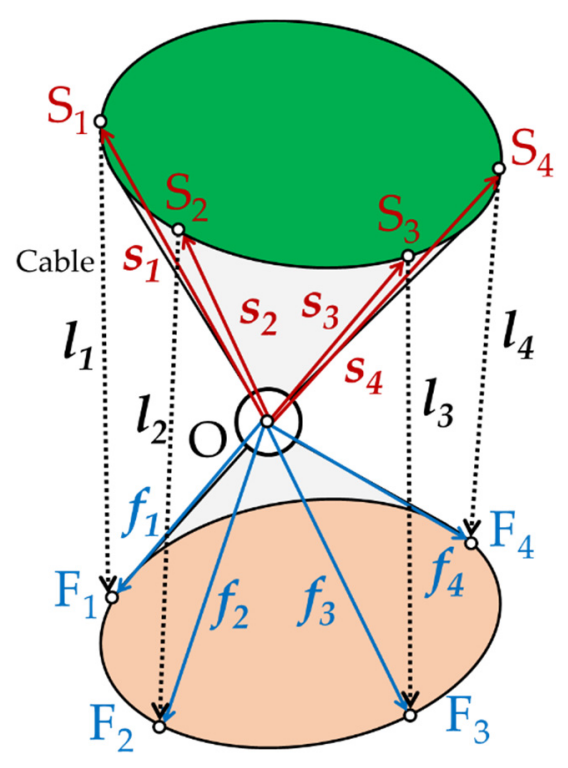

(b)

Figure 3. A kinematic scheme of the proposed solution in Figure 2: (a) with reference frames; (b) with motion variables.

The reference frame $S$ of the shank platform is Oxyz, whereas the reference frame $F$ of the foot platform is Ouvw, as in Figure 3a. A general relative motion of the ankle joint can be described by three consecutive rotations around different axes. Thus, by considering an intrinsic roll-pitch-yaw rotation, the orientation of the foot platform with respect to the shank platform can be expressed as ${ }^{S F} \boldsymbol{R}=\boldsymbol{R}_{z}(\alpha) \boldsymbol{R}_{y}(\beta) \boldsymbol{R}_{x}(\gamma)$. In Figure 3b the $i$ th cable's upper extremity is attached to point $S_{\mathrm{i}}$ of the shank platform, and its lower extremity is attached to the corresponding point $\mathrm{F}_{\mathrm{i}}$ of the foot platform. The following hypotheses are assumed:

- All the cables are always kept in tension during a controlled motion;

- The attachment point of each cable behaves as a spherical joint;

- The varying length of the cable can be modeled as an actuated prismatic joint cable-link with a negligible axial deformation, that can be ensured by a proper cross-section size, material choice (e.g., Dyneema cables), and thanks to the short length of the cables in the proposed application.

The position of the $i$ th cable attachment is ${ }^{s} s_{i}=\left(\begin{array}{lll}s_{i x} & s_{i y} & s_{i z}\end{array}\right)^{T}$ for the shank platform and ${ }^{F} f_{i}=\left(\begin{array}{lll}f_{i x} & f_{i y} & f_{i z}\end{array}\right)^{T}$ for the foot platform. The ankle pose can be described as $x=\left(\begin{array}{lll}\alpha & \beta & \gamma\end{array}\right)^{T}$, where $\alpha, \beta$ and $\gamma$ are the intrinsic rotations around the $z-, y$ - and $x$-axis, as previously defined. The actuation is expressed by $\boldsymbol{q}=\left(\begin{array}{llll}l_{1} & l_{2} & l_{3} & l_{4}\end{array}\right)^{T}$, where $l_{i}$ is the distance between points $S_{\mathrm{i}}$ and $F_{\mathrm{i}}$ of 
each cable, computed as the magnitude of vectors $\boldsymbol{l}_{i}$ in Figure 3. Vectorial loop-closure equations can be written for each cable as

$$
O S_{i}+S_{i} F_{i}+F_{i} O=0
$$

The cable length vector can be computed from (1) as

$$
{ }^{s} l_{i}={ }^{S F} R^{F} f_{i}-{ }^{s} s_{i}
$$

Then, by computing the scalar product of each side of (2) by itself, the length of each cable can be expressed as

$$
l_{i}=\sqrt{{ }^{S} s_{i}^{T S} s_{i}+{ }^{F} f_{i}^{T F} f_{i}-2^{S} s_{i}^{T S F} R^{F} f_{i}}
$$

As reported in Appendix A, the input-output relation can be obtained by deriving Equation (2), as

$$
\dot{\boldsymbol{q}}=\left[\begin{array}{c}
\left({ }^{S F} \boldsymbol{R}^{F} f_{1} \times{ }^{S} u_{1}\right)^{T} \\
\vdots \\
\left({ }^{S F} \boldsymbol{R}^{F} f_{4} \times{ }^{S} u_{4}\right)^{T}
\end{array}\right] \dot{x}
$$

where the $i$ th cable unit vector is defined as $\boldsymbol{u}_{\boldsymbol{i}}$.

\subsection{Static Analysis}

In both rehabilitation and assistance scenarios, the proposed device guides the assisted ankle with a controlled smooth motion, in order to avoid stress, pain or damage to a wearer. Because of the negligible moving mass and the slow speed motion, dynamics and inertial effects can be neglected, and performance evaluation can be computed by using a static model as proposed in the following. When a generic wrench, which can be defined as force $F_{W}$ and moment $M_{W}$, is applied to the foot platform, a static equilibrium can be achieved with a combination of the reaction at the ankle joint, in terms of reaction force $F_{A}$ and reaction moment $M_{A}$, and of cable tension $T$, as in the scheme in Figure 4.

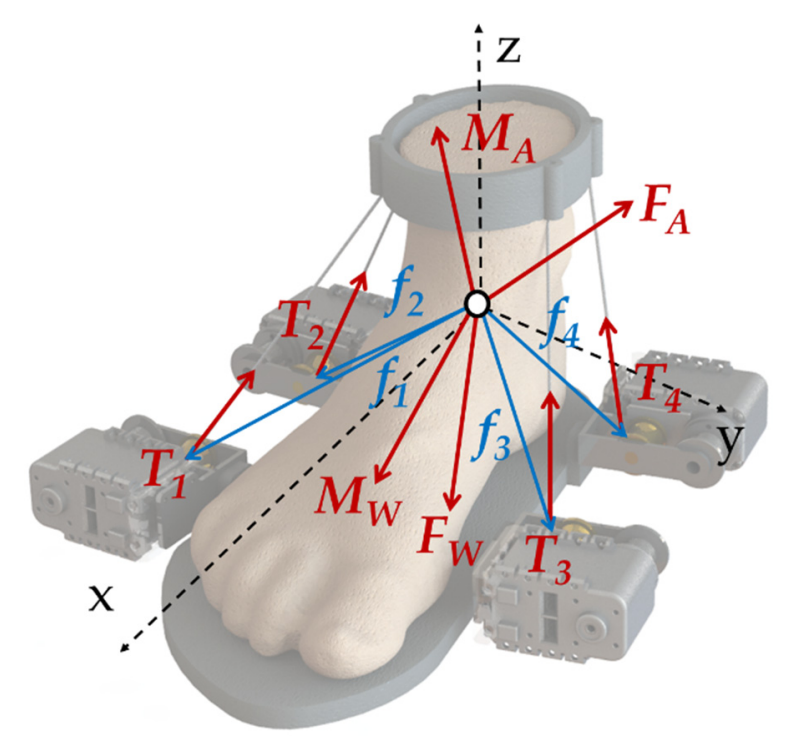

Figure 4. A static model of the CABLEankle mechanism in Figure 2 with conditions for the equilibrium of the ankle assisted by the proposed device. 
Thus, the equilibrium condition to translation in reference frame $S$ is given by

$$
\sum_{i=1}^{n} \boldsymbol{T}_{i}+\boldsymbol{F}_{A}+\boldsymbol{F}_{W}=\mathbf{0}
$$

where $T_{i}$ is the tension in the $i$ th cable, whereas the equilibrium condition to rotation is given by

$$
\sum_{i=1}^{n} f_{i} \times T_{i}+M_{A}+M_{W}=0
$$

By defining cable unit vector $u_{i}$, the tension in the $i$ th cable can be expressed as the product of its magnitude and limb unit vectors $\boldsymbol{u}_{i}$ as $\boldsymbol{T}_{i}=-T_{i} \boldsymbol{u}_{i}$. The actuation vector $\boldsymbol{T}$, with components $\left(\begin{array}{llll}T_{1} & T_{2} & T_{3} & T_{4}\end{array}\right)^{T}$, can be used to rewrite Equations (5) and (6) to get

$$
U^{\mathrm{T}} \cdot \boldsymbol{T}-\boldsymbol{F}_{A}=\boldsymbol{F}_{W}
$$

and the equilibrium condition to rotation can be written as

$$
A^{\mathrm{T}} \cdot \boldsymbol{T}-M_{A}=M_{W}
$$

with $\boldsymbol{U}^{\mathrm{T}}=\left[\begin{array}{llll}\boldsymbol{u}_{1} & \boldsymbol{u}_{2} & \boldsymbol{u}_{3} & \boldsymbol{u}_{4}\end{array}\right]$ and $A^{\mathrm{T}}=\left[\begin{array}{lll}f_{1} \times \boldsymbol{u}_{1} & \cdots & f_{4} \times \boldsymbol{u}_{4}\end{array}\right]$.

Since the ankle joint has been approximated as a spherical joint, it can constrain translational motion only, while reaction moment $\boldsymbol{M}_{\boldsymbol{A}}$ is a null vector within the ankle motion range. Therefore, the full equilibrium condition can be given by

$$
\left[\begin{array}{cc}
\boldsymbol{U}^{\mathrm{T}} & -\mathbf{I}_{3} \\
\boldsymbol{A}^{\mathrm{T}} & \mathbf{0}_{3}
\end{array}\right]\left(\begin{array}{c}
T \\
\boldsymbol{F}_{\boldsymbol{A}}
\end{array}\right)=\left(\begin{array}{c}
\boldsymbol{F}_{W} \\
\boldsymbol{M}_{W}
\end{array}\right)
$$

\subsection{Force Closure}

Unlike rigid links, cables can pull the two bodies together but cannot push. Thus, the results obtained from the kinematics in Equations (3) and (4) and from the static model in Equation (9) cannot be directly applied to a real system. Therefore, the poses where the moving platform is fully constrained by the cables must be determined maintaining positive cable tension as critical condition in constraining the moving platform. Hence, the force-closure workspace is a set of poses whereby resultant cable tensions can sustain an arbitrary external wrench acting on the moving platform, as pointed out in [29].

A general cable-driven parallel mechanism is said to have a force closure in a given pose if and only if any arbitrary external wrench applied at the moving platform can be sustained through appropriate positive tension forces in the cables [30,31]. The condition for force closure is usually described as

$$
\forall\left(\begin{array}{c}
\boldsymbol{F}_{W} \\
\boldsymbol{M}_{\boldsymbol{W}}
\end{array}\right) \in \mathbb{R}^{6} \exists T_{1}, \ldots, T_{4} \in[0, \infty):\left[\begin{array}{cc}
\boldsymbol{U}^{\mathrm{T}} & -\mathbf{I}_{3} \\
\boldsymbol{A}^{\mathrm{T}} & \mathbf{0}_{3}
\end{array}\right]\left(\begin{array}{c}
\boldsymbol{T} \\
\boldsymbol{F}_{\boldsymbol{A}}
\end{array}\right)=\left(\begin{array}{c}
\boldsymbol{F}_{W} \\
\boldsymbol{M}_{\boldsymbol{W}}
\end{array}\right)
$$

A mathematical solution for a standard cable-driven parallel mechanism is well established in scientific literature for the case of $n$-DoF manipulator with $n+1$ cables as reported, for example, in [29-32], but the mechanism under analysis has a hybrid structure with the ankle joint in parallel with the cables. Therefore, a different approach is needed. Without any rigid joint, the force closure would be verified for any of the following conditions:

1. The columns of $\left[\begin{array}{l}\boldsymbol{U}^{\mathrm{T}} \\ \boldsymbol{A}^{\mathrm{T}}\end{array}\right]$ positively span $\mathbb{R}^{6}$; 
2. The convex hull of the column of $\left[\begin{array}{c}U^{\mathrm{T}} \\ A^{\mathrm{T}}\end{array}\right]$ contains the neighborhood of the origin;

3. There does not exist a vector $v \in \mathbb{R}^{6}, v \neq \mathbf{0}$, such as that the scalar product of $v$ and each column of $\left[\begin{array}{c}U^{\mathrm{T}} \\ A^{\mathrm{T}}\end{array}\right]$ is greater than or equal to 0 .

However, the ankle joint is not modeled by those conditions, and it is therefore assumed that the cables must balance any wrench without the contribution of the joint reaction force (which can both pull and push, differently from the cables). Thus, verifying force closure with those conditions would impose unneeded design constraints by not considering the action of the ankle/leg.

Since the two platforms are connected by the ankle joint, the components of the wrench relative to the degrees of freedom that are constrained by the joint can be fully balanced by the reaction of the joint itself, while the cables are used to balance only the reaction moments relative to the idle rotational degrees of freedom. Therefore, the proposed motion assistance device can be said to have force closure in a given pose if and only if any arbitrary external wrench, which is applied at the moving platform and it cannot be balanced by the ankle joint, can be sustained through appropriate (positive) tension forces in the cables. This definition of force closure can be mathematically expressed as

$$
\forall M_{W} \in \mathbb{R}^{3} \exists T_{1}, \ldots, T_{4} \in[0, \infty): A^{\mathrm{T}} \boldsymbol{T}=M_{W}
$$

Therefore, force closure can be verified by any of the following conditions:

1. The columns of $A^{\mathrm{T}}$ positively span $\mathbb{R}^{m}$;

2. The convex hull of the column of $A^{\mathrm{T}}$ contains the neighborhood of the origin;

3. There does not exist a vector $v \in \mathbb{R}^{m}, \boldsymbol{v} \neq \mathbf{0}$, such as that the scalar product of $v$ and each column of $A^{\mathrm{T}}$ is greater than or equal to 0 .

This novel definition of force closure drastically simplifies the solution for a cable-driven rotational joint. A simple example is here reported for a 2D dorsiflexion-plantarflexion motion that is characterized by the simplified geometry in Figure 5. In this example, three possible positions are defined for the cables that will drive the rotation of the ankle around the $Y$-axis, defined by cable attachment points $\mathrm{S}_{1} \mathrm{~F}_{1}, \mathrm{~S}_{2} \mathrm{~F}_{2}$ and $\mathrm{S}_{3} \mathrm{~F}_{3}$, respectively. The main parameters of the example are defined in Table 2.

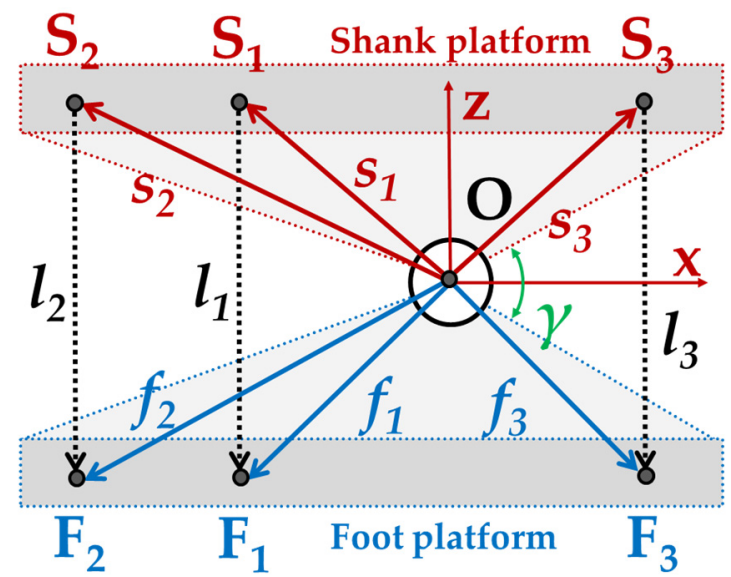

Figure 5. A simplified 2D model of the proposed CABLEankle mechanism for an example of force closure with the proposed formulation. 
Table 2. Parameters of the force-closure example.

\begin{tabular}{cccccccc}
\hline $\boldsymbol{i}$ & $s_{i}$ & $f_{i}$ & $\boldsymbol{u}_{\boldsymbol{i}}$ & $f_{\boldsymbol{i}} \times \boldsymbol{u}_{\boldsymbol{i}}$ \\
\hline 1 & $\left(\begin{array}{lll}-1 & 0 & 1\end{array}\right)^{T}$ & $\left(\begin{array}{lll}-1 & 0 & -1\end{array}\right)^{T}$ & $\left(\begin{array}{lll}0 & 0 & -1\end{array}\right)^{T}$ & $\left(\begin{array}{lll}0 & -1 & 0\end{array}\right)^{T}$ \\
2 & $\left(\begin{array}{lll}-2 & 0 & 1\end{array}\right)^{T}$ & $\left(\begin{array}{lll}-1 & 0 & -1\end{array}\right)^{T}$ & $\left(\begin{array}{lll}0 & 0 & -1\end{array}\right)^{T}$ & $\left(\begin{array}{ccc}0 & -2 & 0\end{array}\right)^{T}$ \\
3 & $\left(\begin{array}{ccc}1 & 0 & 1\end{array}\right)^{T}$ & $\left(\begin{array}{llll}-1 & 0 & -1\end{array}\right)^{T}$ & $\left(\begin{array}{llll}0 & 0 & -1\end{array}\right)^{T}$ & $\left(\begin{array}{ccc}0 & 1 & 0\end{array}\right)^{T}$ \\
\hline
\end{tabular}

When considering a planar motion, the ankle joint has all translational degrees of freedom, thus allowing only the rotation around the $y$-axis. The other rotational degrees of freedom are out of the considered plane and therefore neglected in this example. Three possible combinations of cables are considered to drive the revolute joint, as shown in Table 3. When using Equation (11), the elements of matrix $A^{\mathrm{T}}$ related to the rotation around the $x$-axis and the $z$-axis can be ignored, and by applying the second condition to verify force closure, it is possible to see that the 1D convex hull of the columns of the $1+2$ case does not contain the neighborhood of the origin, while all the other options satisfy the condition. From a physical point of view, it can be noticed that to drive a rigid joint at least a cable on each side is needed to force rotation in both senses. While combinations $1+3$ and $2+3$ easily satisfy this condition, combination $1+2$ cannot, as proved by the example. This calculation can be easily expanded and implemented for the $3 \mathrm{D}$ case (yielding a $3 \times \mathrm{N}$ matrix $A^{\mathrm{T}}$, with $\mathrm{N}>3$, and a convex hull defined by $\mathrm{N}$ points in a 3D space).

Table 3. Results of the force closure example.

\begin{tabular}{|c|c|c|}
\hline Cables & $B_{\text {red }}$ & Force Closure? \\
\hline $1+2$ & $\begin{array}{ll}-1 & -2\end{array}$ & $\mathrm{NO}$ \\
\hline $2+3$ & {$\left[\begin{array}{ll}-2 & 1\end{array}\right.$} & YES \\
\hline $1+3$ & $\begin{array}{ll}-1 & 1\end{array}$ & YES \\
\hline
\end{tabular}

\section{Results}

By using the models developed in Section 2, a target ankle motion can be achieved thanks to the Inverse Kinematic formulation in Equations (3) and (4), whereas the static model in (9) and the force closure condition in (11) can be used as the base for a predictive force control to avoid the wearer from getting injured by excessive ankle load from the proposed CABLEankle operation. In addition, the above kinematics and force analysis can characterize the proposed design in terms of operation performance for a practical implementation of a prototype in a near-future development.

Therefore, the proposed CABLEankle design can be validated on the average motion range of the human ankle, by evaluating its performance on the three motion modes of the ankle, namely dorsiflexion/plantarflexion, inversion/eversion, and adduction/abduction, as illustrated in Figure 1 and with the numerical limits in Table 1. Each motion mode simulates a rotation from the lower to the upper limit of the ankle joint.

\subsection{Kinematics}

The design configuration of the device in Figure 2, for which patent data are given in in [33], is characterized by the geometry in Table 4 with the results of simulated operation in terms of elongation of the actuation cables, tension in the actuation cables, and load on the ankle joint, as in Figures 6-8, respectively. In the tested operations, each cable has a minimum length of $20 \mathrm{~mm}$ and a maximum length of $200 \mathrm{~mm}$. By using the Inverse Kinematic formulation in Equation (3), it is possible to compute the length of each cable for the simulated motion modes with the results that are illustrated in Figure 6 . 
Table 4. Geometrical parameters of the example CABLEankle design in Figure 2 for performance evaluation.

\begin{tabular}{|c|c|c|}
\hline Shank Platform [mm] & Foot Platform [mm] & Joint Motion Limits \\
\hline 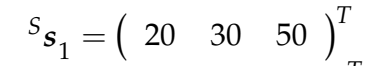 & ${ }^{F} f_{1}=\left(\begin{array}{lll}80 & 75 & -40\end{array}\right)^{T}$ & $\alpha \in\left[-10^{\circ} ; 10^{\circ}\right]$ \\
\hline${ }^{S s_{2}}=\left(\begin{array}{lll}-20 & 30 & 50\end{array}\right)^{T}$ & ${ }^{F} f_{2}=\left(\begin{array}{lll}0 & 60 & -40\end{array}\right)^{T}$ & $\beta \in\left[-50^{\circ} ; 20^{\circ}\right]$ \\
\hline${ }^{S_{s_{3}}}=\left(\begin{array}{lll}20 & -30 & 50\end{array}\right)^{T}$ & ${ }^{F} f_{3}=\left(\begin{array}{lll}10 & 60 & -40\end{array}\right)^{T}$ & $\gamma \in\left[-12^{\circ} ; 12^{\circ}\right]$ \\
\hline${ }^{S_{s_{4}}}=\left(\begin{array}{lll}-20 & -30 & 50\end{array}\right)^{T}$ & ${ }^{F} f_{4}=\left(\begin{array}{lll}90 & 60 & -40\end{array}\right)^{T}$ & $l_{i} \in[20 \mathrm{~mm} ; 200 \mathrm{~mm}]$ \\
\hline
\end{tabular}

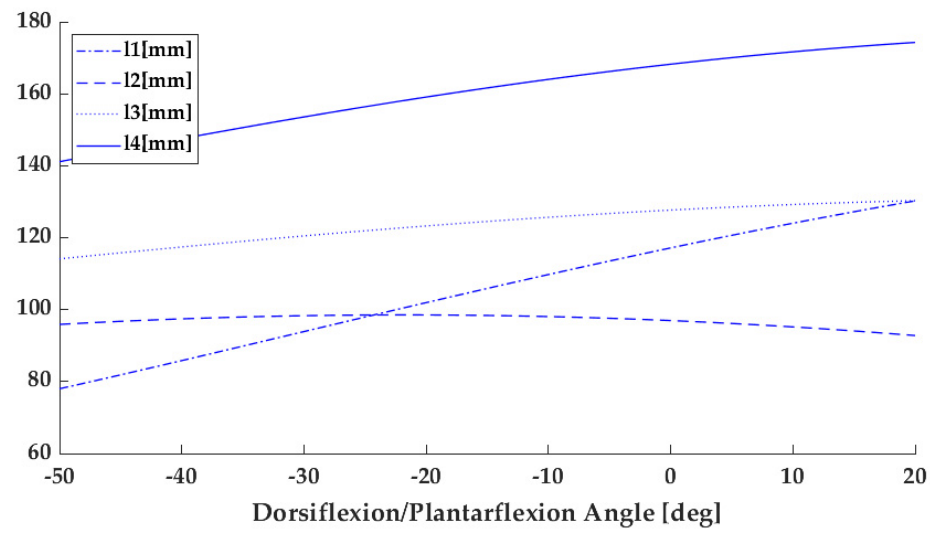

(a)

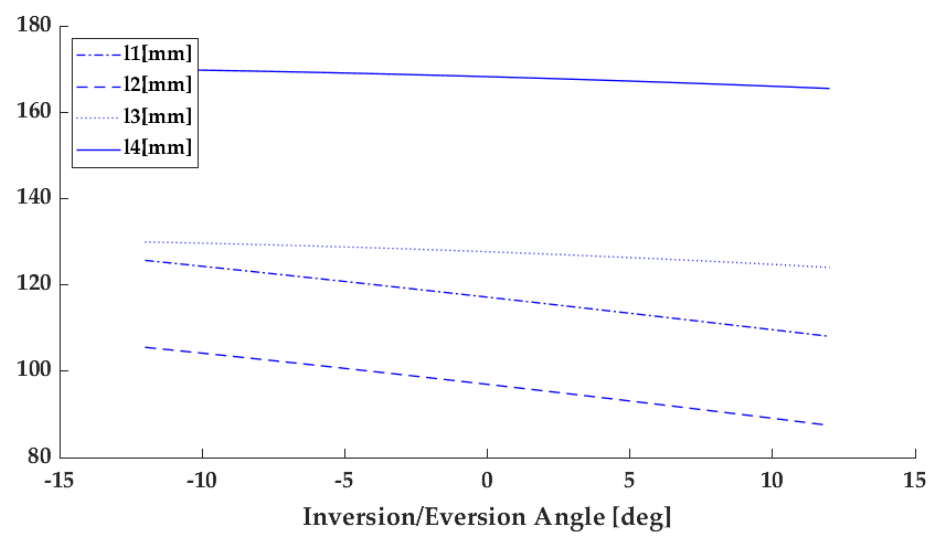

(b)

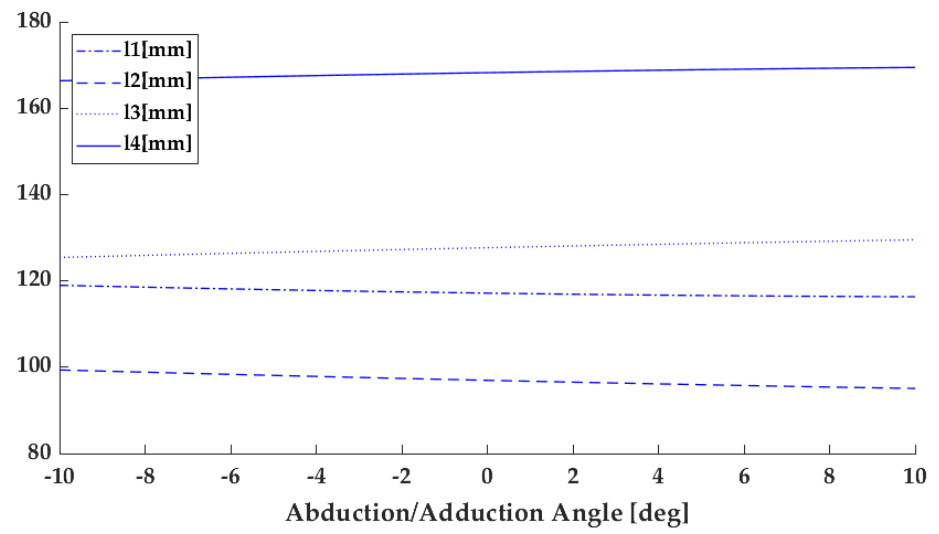

(c)

Figure 6. Computed length of the actuation cables in a simulated full motion cycle: (a) dorsiflexion/plantarflexion; (b) inversion/eversion; (c) abduction/adduction. 


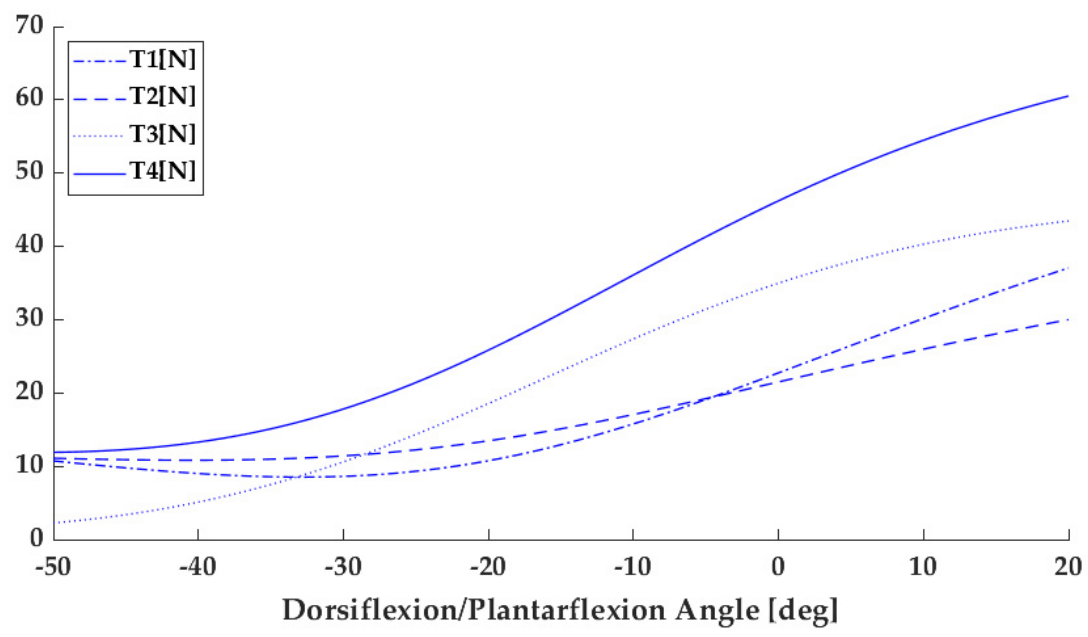

(a)

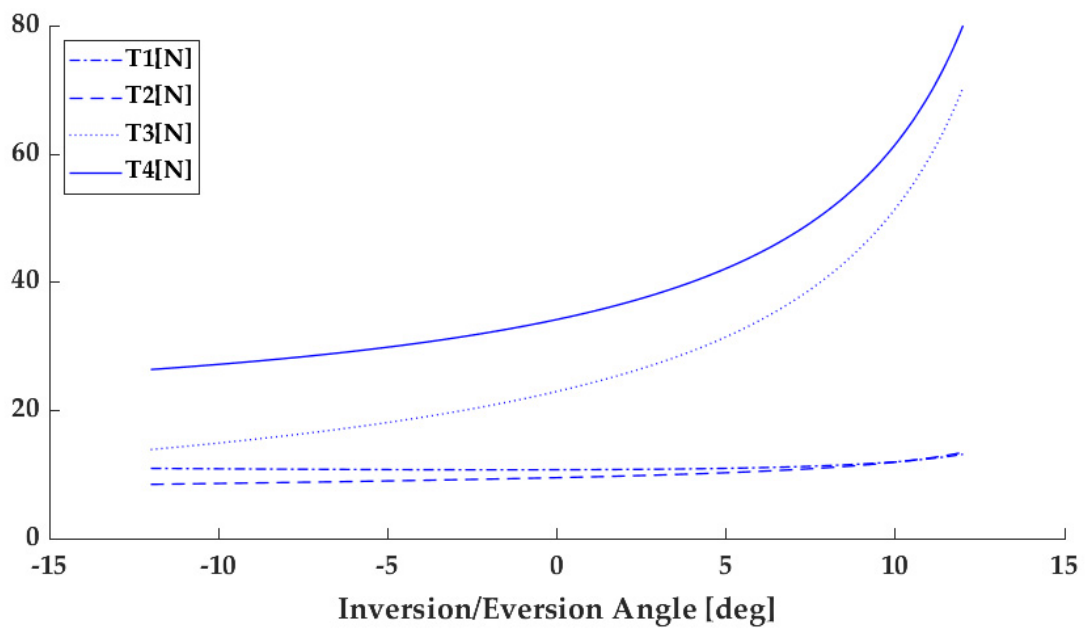

(b)

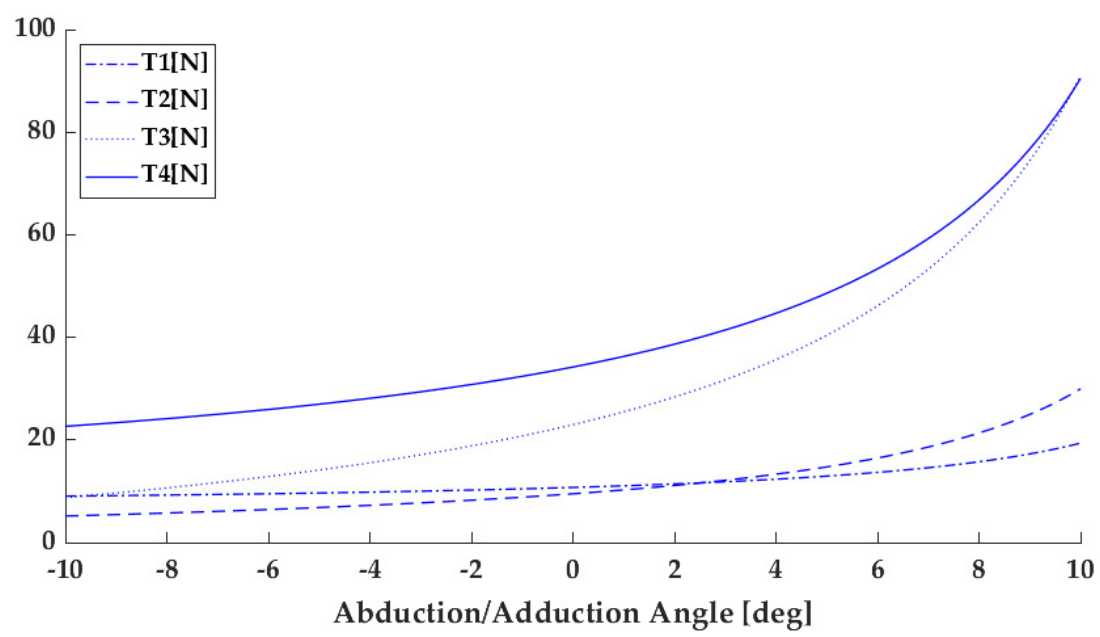

(c)

Figure 7. Computed tension in the actuation cables in a simulated full motion cycle: (a) dorsiflexion/plantarflexion; (b) inversion/Eversion; (c) abduction/adduction. 


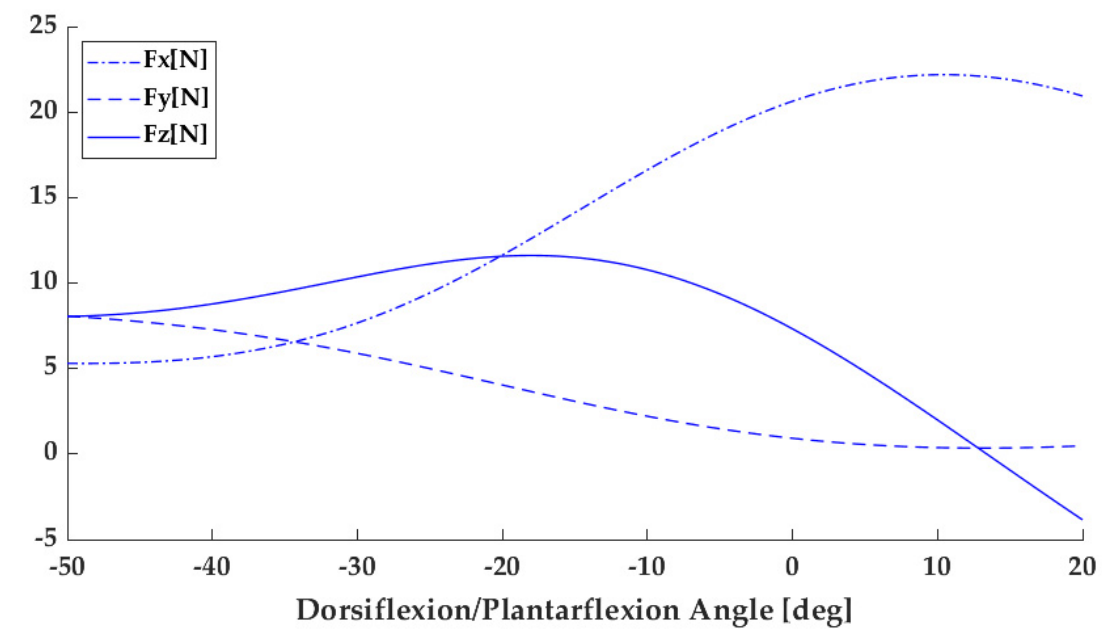

(a)

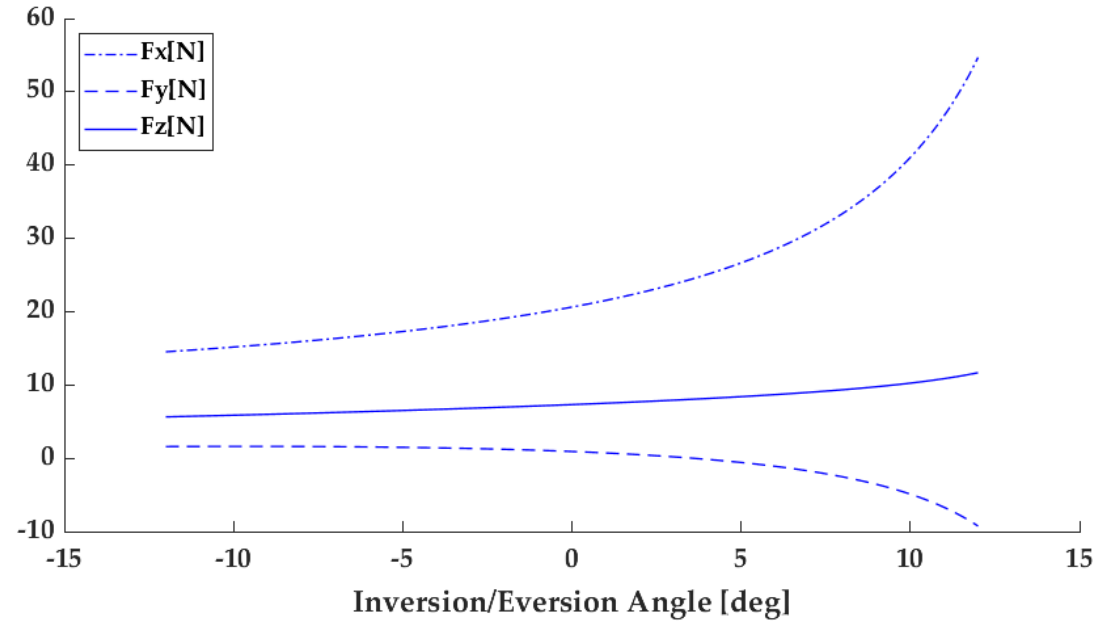

(b)

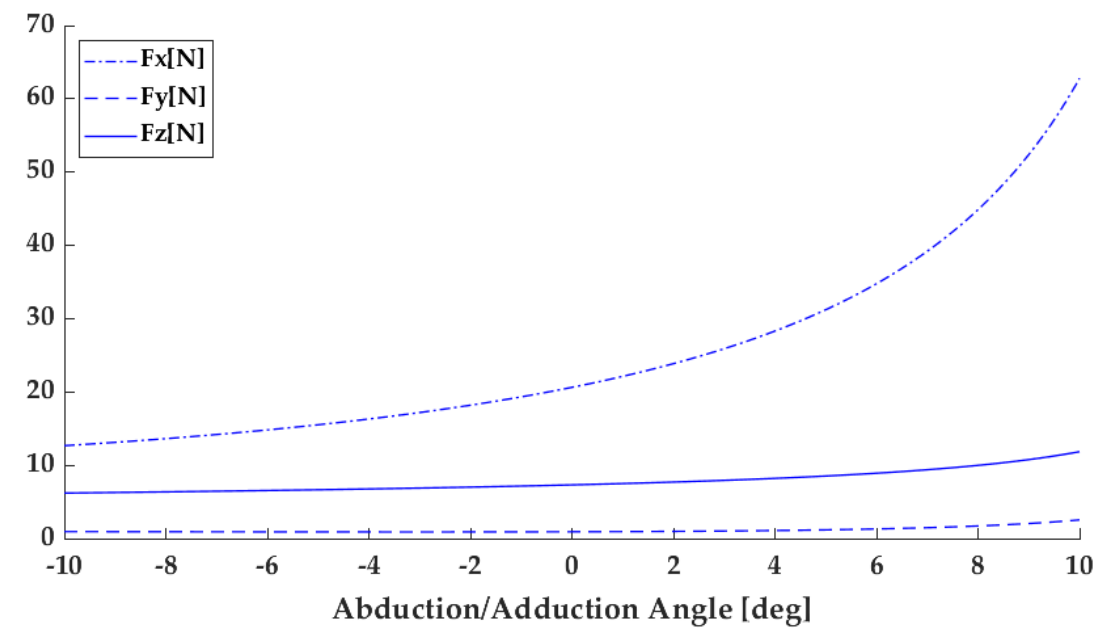

(c)

Figure 8. Computed load on the ankle joint in a simulated full motion cycle: Dorsiflexion/plantarflexion; (b) inversion/eversion; (c) abduction/adduction. 


\subsection{Statics and Force Closure}

The static model of Equation (9) can be used to evaluate both the ankle joint reaction and the tension in the cables. The simulation results have been computed by assuming no external wrench applied onto the foot except for its own weight, with an estimated mass of $1.00 \mathrm{~kg}$ as per an exercise of the ankle on the air. The resulting values of cable tension for the simulated motion modes are reported in Figure 7, whereas the load on the wearer's ankle is shown in Figure 8.

The entire motion range of the ankle, as expressed in Table 1 , is included in the force closure workspace of the proposed cable-driven parallel robot, which is evaluated with the proposed formulation in Equation (11). This condition is essential to ensure that the motion of the ankle can be controlled by the cable-driven mechanism and that no unexpected/uncontrolled motion is allowed.

\subsection{Power Consumption}

By using the simulated motion data, it is also possible to compute the ideal power consumption of the system by assuming negligible losses due to friction and other external factors. For each simulated motion, the total time has been assumed equal to $5.0 \mathrm{~s}$, representing a slow motion from the minimum to the maximum angle for rehabilitation purposes. The instantaneous power consumption $P_{m, i}$ of the $i$ th motor can be estimated as

$$
P_{m, i}=\tau_{m, i} \omega_{m, i}
$$

where $\tau_{m, i}$ is the motor torque, which can be computed by multiplying the tension in the $i$ th cable and the driving pulley diameter, and $\omega_{m, i}$ is the angular velocity of the ith motor, which can be computed from the variation rate of the $i$ th cable $\dot{l}_{i}$, from vector $\dot{q}$ in Equation (4).

For the simulated motion modes, the overall power consumption of the four actuators in the system is characterized by a maximum value of $20 \mathrm{~W}$ when the mechanism moves in the upper limit of its motion range in the inversion/eversion and adduction/abduction motion modes.

\section{Discussion}

The results in Figure 6 demonstrate that the proposed CABLEankle design can operate in the full range of motion of the ankle without reaching joint or device limits. with suitable smooth motion and limited variation of the cable lengths. The largest variation of cable length is computed as $46 \mathrm{~mm}$ for cable 1 in Figure $6 \mathrm{a}$ and the smallest one is of $5 \mathrm{~mm}$ for cable 3 in Figure $6 \mathrm{~b}$.

The values of cable tension that are computed with the proposed static analysis for the simulated motion modes in Figure 7 have a maximum value of 90 N. Thus, they are safely within the maximum value that is allowed by a rotational motor driving the cable. Another key result from the static analysis is the load on the wearer's ankle, as shown in Figure 8. The force acting on the ankle is reported in terms of its cartesian components to emphasize the influence of motion direction, with the component along the $x$-axis of the foot as per Figure 1 being the most significant one, whereas the vertical component along the z-axis shows the largest variability. During the simulated motion, the load reaches a maximum with $60 \mathrm{~N}$, which is well sustainable with a healthy ankle but could be dangerous in the case of rehabilitation of an injured one. In that case of rehabilitation exercise, the inversion/eversion and abduction/adduction motions should be limited to a range less than 10 deg with a suitable ankle load smaller than $30 \mathrm{~N}$. With such a practical range of assisted motion, the tension in the cables are reduced conveniently up to much less than the computed maximum of $90 \mathrm{~N}$.

The low power consumption of the prototype means that the proposed device can be powered by portable batteries. A 10,000 mAh battery, for example, can guarantee up to two hours of continuous functioning, thus removing the need for an external power supply. A power supply with commercial batteries can be integrated in the foot platform, on the shank platform or in a box nearby the device. Thus, the device can include the battery, the motors, a small control board and a Bluetooth module, removing the need for complicated electrical wire routing between different bodies of the robot. 
A portable battery, combined with an estimated weight of $2.0 \mathrm{~kg}$ (of which $0.7 \mathrm{~kg}$ of prototype and $1.3 \mathrm{~kg}$ of battery), makes the proposed design truly lightweight, portable, and user-friendly.

\section{Conclusions}

This paper introduced the CABLEankle, a novel cable-driven mechanism for ankle motion assistance, as inspired by the CADEL design for elbow assistance. The proposed design has been developed as a lightweight wearable solution that is easy to setup and adapt to different patients/users for different kinds of exercises. Furthermore, the lightweight cable-based actuation makes it inherently safe for users because of the negligible mass of the moving bodies and the limited wrench that is used to actuate the system. The novel device has been modeled kinematically and statically in order to evaluate its feasibility in terms of maximum cable tension, load on the ankle joint of the wearer and range of motion. A novel formulation for the evaluation of force closure in hybrid cable-driven mechanisms with both tendons and rigid joints connecting the two platforms has been developed and applied to the proposed mechanism, in order to ensure force closure in its expected motion range. Numerical simulations demonstrate that the mobility of the CABLEankle successfully encompasses the average range of motion of a human ankle joint for daily activities, while exerting a sustainable load on the ankle during the assisted motion.

\section{Patents}

The following patent is a background for the work reported in this manuscript: Russo M., Ceccarelli M., "Dispositivo per assistenza motoria della caviglia (Device for ankle motion assistance)", IT 102020000002863, filed 13/02/2020.

Author Contributions: Conceptualization, M.R. and M.C.; methodology, M.C.; kinematic analysis, M.R.; software and simulation, M.R.; data curation, M.R.; writing—original draft preparation, M.R. and M.C.; writing-review and editing, M.C.; supervision, M.C. Both authors have read and agreed to the published version of the manuscript.

Funding: This research received no external funding.

Conflicts of Interest: The authors declare no conflict of interest.

\section{Appendix A}

This appendix presents the formulation that is used to compute Equation (4). It is possible to formulate the input-output relations by deriving Equation (2) with respect to time, as

$$
\omega_{i} \times{ }^{S} l_{i}+\dot{l}_{i}^{S} u_{i}=\dot{x} \times{ }^{S F} R^{F} b_{i}
$$

where the angular velocity of the $i$ th cable is defined as $\omega_{i}$, and the unit vector of the $i$ th cable is defined as $\boldsymbol{u}_{\boldsymbol{i}}$. The rotation matrix of the foot platform is defined as

$$
{ }^{S F} \boldsymbol{R}=\left[\begin{array}{ccc}
\mathrm{c} \alpha \mathrm{c} \beta & \mathrm{c} \alpha \mathrm{s} \beta \mathrm{s} \gamma-\mathrm{s} \alpha \mathrm{c} \gamma & \mathrm{c} \alpha \mathrm{s} \beta \mathrm{c} \gamma+\mathrm{s} \alpha \mathrm{s} \gamma \\
\mathrm{s} \alpha \mathrm{c} \beta & \mathrm{s} \alpha \mathrm{s} \beta \mathrm{s} \gamma+\mathrm{c} \alpha \mathrm{c} \gamma & \mathrm{s} \alpha \mathrm{s} \beta \mathrm{c} \gamma-\mathrm{c} \alpha \mathrm{s} \gamma \\
-\mathrm{s} \beta & \mathrm{c} \beta \mathrm{s} \gamma & \mathrm{c} \beta \mathrm{c} \gamma
\end{array}\right]
$$

where $\mathrm{c}$ stands for cosine and s for sine. By computing the scalar product of both sides of (A1) by the limb unit vector, the dependency from the angular velocity of the $i$ th cable is removed to get

$$
\dot{l}_{i}=\left({ }^{S F} \boldsymbol{R}^{F} \boldsymbol{b}_{\boldsymbol{i}} \times{ }^{S} \boldsymbol{u}_{i}\right) \cdot \dot{x}
$$

Rewriting (A3) for each parallel limb and assembling the resulting equations in matrix form leads to Equation (4). 


\section{References}

1. Knudson, D. Fundamentals of Biomechanics; Springer Science \& Business Media: Heidelberg, Germany, 2007.

2. Mattacola, C.G.; Dwyer, M.K. Rehabilitation of the ankle after acute sprain or chronic instability. J. Athl. Train. 2002, 37, 413. [PubMed]

3. Chen, E.T.; McInnis, K.C.; Borg-Stein, J. Ankle sprains: Evaluation, rehabilitation, and prevention. Curr. Sports Med. Rep. 2019, 18, 217-223. [CrossRef] [PubMed]

4. Zhang, M.; Davies, T.C.; Xie, S. Effectiveness of robot-assisted therapy on ankle rehabilitation-A systematic review. J. Neuroeng. Rehabil. 2013, 10, 30. [CrossRef] [PubMed]

5. Díaz, I.; Gil, J.J.; Sánchez, E. Lower-limb robotic rehabilitation: Literature review and challenges. J. Robot. 2011, 2011, 759764. [CrossRef]

6. Alvarez-Perez, M.G.; Garcia-Murillo, M.A.; Cervantes-Sánchez, J.J. Robot-assisted ankle rehabilitation: A review. Disabil. Rehabil. Assist. Technol. 2019, 15, 394-408. [CrossRef]

7. Shi, B.; Chen, X.; Yue, Z.; Yin, S.; Weng, Q.; Zhang, X.; Wen, W. Wearable ankle robots in post-stroke rehabilitation of gait: A systematic review. Front. Neurorobot. 2019, 13, 63. [CrossRef]

8. Yoon, J.; Ryu, J.; Lim, K.B. Reconfigurable ankle rehabilitation robot for various exercises. J. Robot. Syst. 2006, 22, S15-S33. [CrossRef]

9. Roy, A.; Krebs, H.I.; Williams, D.J.; Bever, C.T.; Forrester, L.W.; Macko, R.M.; Hogan, N. Robot-aided neurorehabilitation: A novel robot for ankle rehabilitation. IEEE Trans. Robot. 2009, 25, 569-582. [CrossRef]

10. Jamwal, P.K.; Xie, S.; Aw, K.C. Kinematic design optimization of a parallel ankle rehabilitation robot using modified genetic algorithm. Robot. Auton. Syst. 2009, 57, 1018-1027. [CrossRef]

11. Jamwal, P.K.; Hussain, S.; Ghayesh, M.H.; Rogozina, S.V. Impedance control of an intrinsically compliant parallel ankle rehabilitation robot. IEEE Trans. Ind. Electron. 2016, 63, 3638-3647. [CrossRef]

12. Saglia, J.A.; Tsagarakis, N.G.; Dai, J.S.; Caldwell, D.G. Control strategies for patient-assisted training using the ankle rehabilitation robot (ARBOT). IEEE/ASME Trans. Mechatron. 2012, 18, 1799-1808. [CrossRef]

13. Sung, E.; Slocum, A.H.; Ma, R.; Bean, J.F.; Culpepper, M.L. Design of an ankle rehabilitation device using compliant mechanisms. J. Med. Devices 2011, 5, 011001. [CrossRef]

14. Lin, C.C.; Ju, M.S.; Chen, S.M.; Pan, B.W. A specialized robot for ankle rehabilitation and evaluation. J. Med. Biol. Eng. 2008, 28, 79-86.

15. Zhang, M.; McDaid, A.; Veale, A.J.; Peng, Y.; Xie, S.Q. Adaptive trajectory tracking control of a parallel ankle rehabilitation robot with joint-space force distribution. IEEE Access 2019, 7, 85812-85820. [CrossRef]

16. Chang, T.C.; Zhang, X.D. Kinematics and reliable analysis of decoupled parallel mechanism for ankle rehabilitation. Microelectron. Reliab. 2019, 99, 203-212. [CrossRef]

17. Nurahmi, L.; Caro, S.; Solichin, M. A novel ankle rehabilitation device based on a reconfigurable 3-RPS parallel manipulator. Mech. Mach. Theory 2019, 134, 135-150. [CrossRef]

18. Cafolla, D.; Russo, M.; Carbone, G. Design and Validation of an Inherently-Safe Cable-Driven Assisting Device. Int. J. Mech. Control 2018, 19, 23-32.

19. Cafolla, D.; Russo, M.; Carbone, G. CUBE, a cable-driven device for limb rehabilitation. J. Bionic Eng. 2019, 16, 492-502. [CrossRef]

20. Ceccarelli, M.; Ferrara, L.; Petuya, V. Design of a Cable-Driven Device for Elbow Rehabilitation and Exercise. In Interdisciplinary Applications of Kinematics; Springer: Cham, Switzerland, 2019; pp. 61-68.

21. Pott, A.; Bruckmann, T. (Eds.) Cable-Driven Parallel Robots: Proceedings of the 4th International Conference on Cable-Driven Parallel Robots; Springer: Cham, Switzerland, 2019.

22. Jamwal, P.K.; Aw, K.C.; Xie, S.Q.; Tsoi, Y.H. Multi-criteria optimal design of cable driven ankle rehabilitation robot. In Mobile Robots-State of the Art in Land, Sea, Air, and Collaborative Missions; INTECH Open Access Publisher: London, UK, 2009.

23. Shahrol, M.N.; Basah, S.N.; Basaruddin, K.S.; Ahmad, W.K.W.; Ahmad, S.A. Modelling of a Cable-driven Ankle Rehabilitation Robot. J. Telecommun. Electron. Comput. Eng. 2018, 10, 53-59.

24. Russo, M.; Ceccarelli, M. A wearable device for ankle motion assistance. In Advances in Italian Mechanism Science: Mechanisms and Machine Science 91; Springer: Cham, Switzerland, 2020; in print.

25. Brockett, C.L.; Chapman, G.J. Biomechanics of the ankle. Orthop. Trauma 2016, 30, 232-238. [CrossRef]

26. Roaas, A.; Andersson, G.B. Normal range of motion of the hip, knee and ankle joints in male subjects, 30-40 years of age. Acta Orthop. Scand. 1982, 53, 205-208. [CrossRef] [PubMed] 
27. Hemmerich, A.; Brown, H.; Smith, S.; Marthandam, S.S.K.; Wyss, U.P. Hip, knee, and ankle kinematics of high range of motion activities of daily living. J. Orthop. Res. 2006, 24, 770-781. [CrossRef] [PubMed]

28. Leirós-Rodríguez, R.; García-Soidán, J.L.; Romo-Pérez, V. Analyzing the use of accelerometers as a method of early diagnosis of alterations in balance in elderly people: A systematic review. Sensors 2019, 19, 3883.

29. Gouttefarde, M.; Gosselin, C.M. Analysis of the wrench-closure workspace of planar parallel cable-driven mechanisms. IEEE Trans. Robot. 2006, 22, 434-445. [CrossRef]

30. Gouttefarde, M.; Merlet, J.P.; Daney, D. Wrench-feasible workspace of parallel cable-driven mechanisms. In Proceedings of the 2007 IEEE International Conference on Robotics and Automation, Roma, Italy, 10-14 April 2007; IEEE: Piscataway, NJ, USA, 2007; pp. 1492-1497.

31. Pham, C.B.; Yeo, S.H.; Yang, G.; Kurbanhuse, M.S.; Chen, I.M. Force-closure workspace analysis of cable-driven parallel mechanisms. Mech. Mach. Theory 2006, 41, 53-69. [CrossRef]

32. Lim, W.B.; Yang, G.; Yeo, S.H.; Mustafa, S.K. A generic force-closure analysis algorithm for cable-driven parallel manipulators. Mech. Mach. Theory 2011, 46, 1265-1275. [CrossRef]

33. Russo, M.; Ceccarelli, M. Device for Ankle Motion Assistance. Italian Patent 102020000002863, 13 February 2020.

(C) 2020 by the authors. Licensee MDPI, Basel, Switzerland. This article is an open access article distributed under the terms and conditions of the Creative Commons Attribution (CC BY) license (http://creativecommons.org/licenses/by/4.0/). 